Pacific Journal of Mathematics

NONLINEAR HOLOMORPHIC SEMIGROUPS

mas Lee Hayden and Frank Jones Massey 


\title{
NONLINEAR HOLOMORPHIC SEMIGROUPS
}

\author{
T. L. Hayden and F. J. Massey, III
}

Conditions are given on a nonlinear operator $A$ in a Banach space $X$ under which the semigroup, $S(t)$, generated by $-A$ has the property that $S(t) x$ is analytic in $t$ for $|\arg t|<\theta$ for each fixed $x \in \operatorname{cl}(D(A))$. Analyticity in $t$ of solutions of $u^{\prime}+T u=F u$ where $-T$ generates a linear holomorphic semigroup in $X$ and $F$ maps $D\left(T^{\alpha}\right)$ analytically into $X$ for some $\alpha<1$ is also established. These results are applied to establish analyticity in $t$ of solutions to $\partial u / \partial t+L u+\beta(u)=0$ where $\beta: R \rightarrow R$ is real analytic, monotone increasing and $\beta(0)=0$, and $L$ is a second order elliptic operator.

1. Introduction. Hille and Yosida proved that if $A$ is a densely defined linear operator on a Banach space $X$ such that, for $\lambda>0$, $I+\lambda A$ is an isomorphism from $D(A)$ onto $X$ and $(I+\lambda A)^{-1}$ is a contraction, then $-A$ generates a strongly continuous semigroup $\{S(t): t \geqq 0\}$ of contractions on $X$. If $X$ is a complex Banach space and the above conditions hold for $|\arg \lambda|<\theta$, instead of just for $\lambda>0$, then $S(t)$ has an analytic extension in $t$ to the sector $|\arg t|<\theta$. These holomorphic semigroups have a smoothing property, namely $S(t)$ maps $X$ into $D(A)$ for $t \neq 0$ so that $u(t)=S(t) x$ is a solution to $u^{\prime}(t)+A u(t)=0, u(0)=x$ for any initial data $x \in X$. For the linear theory of semigroups see Yosida [24], Kato [12], and HillePhillips [11].

A number of authors (see Kōmura [15, 16], Kato [13, 14], Crandall and Pazy [6], Brezis [2], Crandall and Liggett [5], and the references listed there) have generalized the theory of semigroups to nonlinear operators. They have shown that if $A \subset X \times X$ is a (multivalued) nonlinear operator such that, for sufficiently small $\lambda>0,(I+\lambda A)^{-1}$ is a contraction and the range of $(I+\lambda A)$ contains $\operatorname{cl}(D(A))$, the closure of the domain of $A$, then $-A$ generates a strongly continuous semigroup $\{S(t): t \geqq 0\}$ on $\operatorname{cl}(D(A))$. In the case when $X$ is a Hilbert space, Kōmura [16] has given conditions under which $S(t)$ extends analytically to a sector $|\arg t|<\theta$. Brezis [2] has shown that if $A=\partial \varphi$ is the subdifferential of a lower semicontinuous, convex functional on a Hilbert space then the semigroup $\{S(t)\}$ generated by $-A$ has a regularizing property similar to the linear case, namely $S(t)$ maps cl $(D(A))$ into $D(A)$ for $t>0$.

In this paper (\$2) we give an extension of Kömura's result to the case where $X$ is a Banach space by establishing conditions under which $S(t)$ extends analytically to $|\arg t|<\theta$. These conditions also imply $S(t)$ maps cl $(D(A))$ into $D(A)$ for $t \neq \theta$; in other words, $S(t)$ 
has a smoothing action.

In $\S 3$ we establish local analyticity in $t$ of solutions, $u(t)$, of equations of the form $d u / d t+T u=F u$ where $-T$ is the generator of a linear analytic semigroup in a Banach space $X$ and $F$ maps $D\left(T^{\alpha}\right)$ analytically into $X$ for some $\alpha<1$. We use the integral equation approach developed by Sobolevskii [23], and Fujita and Kato [9]. In $\S 4$ we give applications to semilinear parabolic equations.

We wish to thank H. Brezis, M. Crandall, D. Henry, T. Kato, J. W. Neuberger and A. Pazy for their many valuable conversations and useful suggestions concerning this work.

2. A class of holomorphic nonlinear semigroups. In the following $X$ is a complex Banach space. Let $C \subset X$, and $\Sigma_{\theta}=\{z \in$ $C:|\arg z|<\theta, z \neq 0\}$ be an open sector in the complex plane. A holomorphic semigroup on $C$ is a function $S$ on $\Sigma_{\theta} \cup\{0\}$ such that $S(z)$ maps $C$ into $C$ for each $z \in \Sigma_{\theta} \cup\{0\} ; S(z+w)=S(z) S(w)$ for $z, w \in \Sigma_{\theta} \cup\{0\}$; and, for $x \in C, S(z) x$ is a holomorphic function of $z \in \Sigma_{\theta}$ with $S(z) x \rightarrow S(0) x=x$ as $z \rightarrow 0$ and $z \in \Sigma_{\theta}$. If there is also a real number $\omega$ such

$$
\|S(z) x-S(z) y\| \leqq e^{\omega|z|}\|x-y\|,
$$

$x, y \in C, z \in \Sigma_{\theta}$, we will write $S \in \mathscr{H}_{\omega, \theta}(C)$. Note that we do not require $S(z)$ to be holomorphic for fixed $z$ as did Kōmura [16]. Kōmura noted that a contraction mapping which is holomorphic on all of a complex Banach space must be the translate of a linear operator (a consequence of Liouville's theorem). Hence we wish to avoid the hypothesis that $S(z)$ be a holomorphic map.

The generator, $A$, of a nonlinear semigroup is, in general, a "multivalued" operator which is regarded as a subset of $X \times X$. For such operators we use the notation and definitions of Crandall and Liggett [5, page 266].

THEOREM 2.1. Let $A \subset X \times X, \omega, \theta, \varepsilon$ be real numbers such that $e^{i \varphi} A+\omega I$ is accretive for $|\varphi|<\theta$ and $R(I+\lambda A) \supset \operatorname{cl}(D(A))$ for $|\arg \lambda|<\theta$ and $|\lambda|<\varepsilon$. Let $J_{\lambda}=(I+\lambda A)^{-1}$ and suppose, for $x \in D(A)$ and $n$ a positive integer, the map $\lambda \mapsto J_{\lambda}^{n} x$ is a holomorphic function of $\lambda$ for $|\arg \lambda|<\theta,|\lambda|<\min \left(\varepsilon,|\omega|^{-1}\right)$. Then

$$
\lim _{n \rightarrow \infty} J_{z \mid n}^{n} x \equiv S(z) x
$$

exists for $x \in \operatorname{cl}(D(A))$ and $z \in \Sigma_{\theta}$ and $S \in \mathscr{H}_{\omega, \theta}(\operatorname{cl}(D(A))$. If, in addition, $A$ is a closed subset of $X \times X$ then for each $x \in \operatorname{cl}(D(A))$ and $z \in \Sigma_{\theta}$, we have $S(z) x \in D(A)$ and $-(d / d z) S(z) x \in A S(z) x$. 
Proof. Let $K_{\alpha, \varphi}=\left(I+\alpha e^{i \varphi} A\right)^{-1}$ be the resolvent of $e^{i \varphi} A$. For $|\varphi|<\theta$, the operator $e^{i \varphi} A$ satisfies the hypotheses of Theorem 1 of Crandall and Liggett [5], so $\lim K_{t \mid n, \varphi}^{n} x \equiv T_{\varphi}(t) x$ exists for $x \in \operatorname{cl}(D(A))$, $t \geqq 0$, and $\left\{T_{\varphi}(t): t \geqq 0\right\}$ is a (strongly continuous) semigroup with each $T_{\varphi}(t)$ Lipschitz with constant $e^{\omega t}$. Since $J_{\lambda}=K_{|\lambda|, \arg \lambda}$, it follows that the limit (2.2) exists, $S(z) x=T_{\arg z}(|z|) x$, and $S(z)$ satisfies (2.1) for $x, y \in \operatorname{cl}(D(A))$.

Now let $x \in D(A)$. Applying the inequalities (ii) and (iii) on p. 268 of [5] to $e^{i \varphi} A$, we get $\left\|K_{t \mid n, \varphi}^{n} x-x\right\| \leqq t\left(1-t n^{-1}|\omega|\right)^{-n}\left|e^{i \varphi} A x\right|, t \geqq 0$, $t|\omega|<n$. Substituting $t=|z|, \phi=\arg z$, and using $J_{z}=K_{|z| \text {,arg } z}$, and the fact that $(1-a / n)^{-n} \leqq e^{|a|}, a \in R$, we obtain $\left\|J_{z \mid n}^{n} x-x\right\| \leqq$ $|z| e^{|z||\omega|}|A x|,|\arg z|<\theta,|z \omega|<n$. Thus when $z$ is restricted to lie in a bounded subset of $\Sigma_{\theta}$, the sequence $\left\{J_{z \mid n}^{n} x\right\}$ is a uniformly bounded sequence of holomorphic functions of $z$ which converge pointwise to $S(z) x$. It follows (see [11], p. 104) that $S(z) x$ is holomorphic in $z$ and $\| S(z) x-x|| \leqq|z| e^{|z||\omega|}|A x|$. In particular, $S(z) x \rightarrow x$ as $z \rightarrow 0$.

Now let $x \in \operatorname{cl}(D(A))$ and choose $\left\{x_{n}\right\} \subset D(A)$ with $x_{n} \rightarrow x$. Then $\left\{S(z) x_{n}\right\}$ is a sequence of functions holomorphic on $\Sigma_{\theta}$ and continuous at $z=0$. If $z$ is restricted to lie in a bounded subset of $\Sigma_{\theta} \cup\{0\}$ then the $S(z)$ are Lipschitz with constant independent of $z$ and, hence, $\left\{S(z) x_{n}\right\}$ converges uniformly to $S(z) x$. Thus $S(z) x$ is holomorphic on $\Sigma_{\theta}$ and continuous at $z=0$.

In order to show the semigroup property, let $w \in \Sigma_{\theta}$ be fixed and $\varphi=\arg w$. If $\left\{T_{\varphi}(t): t \geqq 0\right\}$ is the semigroup generated by $-e^{i \varphi} A$ then $S\left(t e^{i \varphi}\right)=T_{\varphi}(t), t \geqq 0$. By Crandall and Liggett, $T_{\varphi}(t)$ is a semigroup for real $t$, so $S\left(t e^{i \varphi}+\tau e^{i \varphi}\right)=S\left(t e^{i \varphi}\right) S\left(\tau e^{i \varphi}\right)$. Thus $\mathbf{S}(z+w)=$ $S(z) S(w)$ for $z=t w, t \geqq 0$. If $x \in \operatorname{cl}(D(A))$ then $S(z+w) x$ and $S(z) S(w) x$ are holomorphic functions of $z \in \Sigma_{\theta}$ which agree on the ray $z=t w, t \geqq 0$. By the identity theorem for holomorphic functions $S(z+w) x=S(z) S(w) x$ for all $z$.

In the real case (see [5]) a strong solution to the Cauchy problem

$$
0 \in d u / d t+A u, \quad 0 \leqq t \leqq T, \quad u(0)=x,
$$

is a function $u:[0, T] \rightarrow X$ so that (i) $u$ is continuous, (ii) $u$ is the indefinite integral of a function which is strongly integrable on compact subsets of $(0, T)$, (iii) $u(0)=x$ and (iv) $u^{\prime}(t) \in-A u(t)$ for a.e. $t$ in $(0, T)$.

Crandall and Liggett, and Miyadera [20] have shown the following result. Let $B$ be closed in $X \times X, B+\omega I$ accretive for some real number $\omega, R(I+t B) \supset \mathrm{cl}(D(B))$ for sufficiently small $t>0$, and for $x \in \operatorname{cl}(D(B))$ let $T(t) x=\lim (I+(t / n) B)^{-n} x$ be the semigroup generated by $-B$. Then if $x \in \operatorname{cl}(D(B))$ and $T(t) x$ is strongly differentiable at 
$t_{0}>0$, with $y=(d / d t) T\left(t_{0}\right) x$, then $\left[T\left(t_{0}\right) x,-y\right] \in B$. Then using the fact that for $x \in D(B), S(t) x$ is Lipschitz continuous on bounded sets of $t$, they are able to conclude that if $S(t) x$ is differentiable a.e. then $u=S(t) x$ is a strong solution of (2.3).

In our case, since we have shown that $S(z) x$ is a holomorphic function for $x \in \mathrm{cl}(D(A))$, it is immediate that $S(z) x$ can be recovered as the indefinite integral of an analytic function along a ray.

To finish the details of the proof, let $A$ be closed, $x \in \operatorname{cl}(D(A))$, $z \in \Sigma_{\theta}$ with $\varphi=\arg z$, and $\left\{T_{\varphi}(t) ; t \geqq 0\right\}$ be the semigroup generated by $-e^{i \varphi} A$ so that $S\left(t e^{i \varphi}\right)=T_{\varphi}(t), t \geqq 0$. If $x \in \operatorname{cl}(D(A))$ then $u(z)=$ $S(z) x$ is holomorphic for $z \in \Sigma_{\theta}$ which implies that $v(t)=T_{\varphi}(t) x$ is differentiable for $t>0$ and $v^{\prime}(t)=e^{i \varphi} u^{\prime}\left(t e^{i \varphi}\right)$.

Since $-e^{i \varphi} A$ is closed, it follows from the above results of Crandall and Liggett that $-v^{\prime}(t) \in e^{i \varphi} A v(t)$. Hence $-u^{\prime}\left(t e^{i \varphi}\right) \in A u\left(t e^{i \varphi}\right)$, and together with the comment on holomorphy of $S(t) x$ for $x \in \operatorname{cl}(D(A))$, we have established a strong solution to the Cauchy problem for $x \in \operatorname{cl}(D(A))$.

REMARK. We will show in an example that $J_{\lambda}$ may not be defined on an open set, so that $J_{\lambda}$ is certainly not a holomorphic map in general. However in case $J_{\lambda}$ is a holomorphic map, then the hypothesis $J_{\lambda}^{n} x$ is a holomorphic function of $\lambda$ for all $n$ is satisfied. We may argue as follows. First since $J_{\lambda}$ is locally Lipschitz, both Kömura [16] and Neuberger [21] have established that $J_{\lambda} x$ is holomorphic in $\lambda$ when $J_{\lambda}$ is a holomorphic map. Next let $g\left(\lambda_{1}, \lambda_{2}, \cdots, \lambda_{n}\right)=J_{\lambda_{1}} \cdot J_{\lambda_{2}} \cdot J_{\lambda_{3}} \ldots J_{\lambda_{n}} x$. Then for fixed $\lambda_{2}, \lambda_{3}, \cdots, \lambda_{n}, g$ is holomorphic in $\lambda_{1}$. If $\lambda_{1}, \lambda_{3}, \cdots, \lambda_{n}$ are fixed, then $J_{\lambda_{2}} \cdot J_{\lambda_{3}} \cdots J_{\lambda_{n}}$ is holomorphic in $\lambda_{2}$ and therefore when composed with the holomorphic map $J_{\lambda_{1}}, g$ is holomorphic in $\lambda_{2}$ and so forth. Hence, as is well known [11], p. 107, $g(\lambda, \lambda, \lambda, \cdots)$ is a holomorphic function of $\lambda$.

Example. Let $\beta: K \rightarrow C$ be continuous where $K$ is the closure of an open, convex set $U \subset C$. Suppose $0 \in K, \beta(0)=0$ and $\beta$ is analytic on $U$. Assume there is $\theta>0$ such that $\left|\arg \beta^{\prime}(z)\right| \leqq \pi / 2-\theta$, $z \in U$. Finally suppose there is $\varepsilon<0$ such that for $|\arg \lambda|<\theta$, $|\lambda|<\varepsilon$, one has $(I+\lambda \beta)(K) \supset K$ and $(I+\lambda \beta)(U) \supset U$. Here $I(z)=z$ is the identity map on $C$.

Let $X=L^{p}(\Omega ; C)$ where $\Omega$ is any measure space and $1 \leqq p \leqq \infty$. Let $D(A)=\{u \in X: u(x) \in K$ a.e. and $\beta(u) \in X\}$, where $\beta(u)$ is the composition of $\beta$ and $u$. Let $A u=\beta(u)$ for $u \in D(A)$. We shall show that $A$ satisfies the hypotheses of Theorem 2.1 with $\omega=0$ and $\theta, \varepsilon$ as above. 
The hypothesis $\left|\arg \beta^{\prime}(z)\right| \leqq \pi / 2-\theta, z \in U$, implies $e^{i \varphi} \beta$ is accretive for $|\varphi|<\theta$. In particular $I+\lambda \beta$ is one-to-one and $(I+\lambda \beta)^{-1}$ is a contraction for $|\arg \lambda|<\theta$. Let $S=\{\lambda \in C:|\arg \lambda|<\theta,|\lambda|<\varepsilon\}$. The assumption that $(I+\lambda \beta)(K) \supset K, \lambda \in S$, implies the function $j(w, \lambda)=(I+\lambda \beta)^{-1}(w)$ is well defined for $w \in K, \lambda \in S$. It is a contraction in $w$ for fixed $\lambda$. Since $\beta$ is analytic on $U$ and $(I+\lambda \beta)(U) \supset U$, the implicit function theorem implies $j: U \times S \rightarrow U$ is analytic. Since $\beta(0)=0$ we have $j(0, \lambda)=0$. Since $j(\cdot, \lambda)$ is a contraction we have $|j(w, \lambda)| \leqq|w|$.

Let $j^{1}(w, \lambda)=j(w, \lambda), w \in K, \lambda \in S$ and $j^{n}(w, \lambda)=j\left(j^{n-1}(w, \lambda), \lambda\right)$, $w \in K, \lambda \in S, n \geqq 2$. Since $j(w, \lambda)$ is a contraction in $w$, it follows that $j^{n}(w, \lambda)$ is a contraction in $w$ for fixed $\lambda$. Since $j: U X S \rightarrow U$ is analytic, it follows that $j^{n}: U X S \rightarrow U$ is analytic. We claim that $j^{n}(w, \lambda)$ is analytic in $\lambda$ for fixed $w$, even if $w \in K$. To see this, choose a sequence $\left\{w_{m}\right\} \subset U$ with $w_{m} \rightarrow w$. Then $\left\{j^{n}\left(w_{m}, \lambda\right)\right\}$ is a sequence of functions each analytic in $\lambda$ and $j^{n}\left(w_{m}, \lambda\right) \rightarrow j^{n}(w, \lambda)$ uniformly in $\lambda$ since $j^{n}(w, \lambda)$ is a contraction in $w$. It follows that $j^{n}(w, \lambda)$ is analytic in $\lambda$. Finally we note that $\left|j^{n}(w, \lambda)\right| \leqq|w|$ since $|j(w, \lambda)| \leqq|w|$.

Now consider the operator $A$. We have $v=(I+\lambda A) u$ if and only if $v(x)=(I+\lambda \beta)(u(x))$ a.e. If $|\arg \lambda|<\theta$ then $I+\lambda \beta$ is $1-1$ so $v=(I+\lambda A) u$ is equivalent to $u(x)=(I+\lambda \beta)^{-1}(v(x))$ a.e. In particular $I+\lambda A$ is $1-1$ and $J_{\lambda} \equiv(I+\lambda A)^{-1}$ is contraction. It follows that $e^{i \varphi} A$ is accretive for $|\varphi|<\theta$.

To show $\operatorname{cl}(D(A)) \subset R(I+\lambda A)$, note that $\operatorname{cl}(D(A)) \subset F$ where $F=\{v \in X: v(x) \in K$ a.e. $\}$. The assumption $K \subset(I+\lambda \beta)(K), \lambda \in S$ and the definition of $j$ implies that $F \subset R(I+\lambda A)$ for $\lambda \in S$, and $J_{\lambda} v(x)=$ $j(v(x), \lambda), v \in F, \lambda \in S$.

To show $J_{\lambda}^{n} v$ is analytic in $\lambda$ for fixed $v \in F$, note that $J_{\lambda}^{n} v(x)=$ $j^{n}(v(x), \lambda)$. It follows from $\left|j^{n}(v(x), \lambda)\right| \leqq|v(x)|$ and the Cauchy integral formula that

$$
\begin{gathered}
\left|j_{\lambda}^{n}(v(x), \lambda)\right| \leqq|v(x)| \operatorname{dist}(\lambda, \partial S) \\
\left|j_{\lambda \lambda}^{n}(v(x), \lambda)\right| \leqq|v(x)|[\operatorname{dist}(\lambda, \partial S)]^{2}
\end{gathered}
$$

where $j_{\lambda}^{n}=\partial j^{n} / \partial \lambda, j_{\lambda \lambda}^{n}=\partial^{2} j^{n} / \partial \lambda^{2}$. In the case $1 \leqq p<\infty$ in order to show $J_{\lambda}^{n} v$ is analytic in $\lambda$ it suffices to show weak analyticity, i.e. $(d / d \lambda) \int_{\Omega} j^{n}(v(x), \lambda) \overline{w(x)} d x=\int_{\Omega} j_{\lambda}^{n}(v(x), \lambda) \overline{w(x)} d x$ for all $w \in L^{q}(\Omega), p^{-1}+$ $q^{-1}=1$. This is true because $j^{n}(v(x), \lambda)$ is analytic in $\lambda$ for fixed $x$, and the estimate (2.4) implies that differentiation under the integral sign is valid. In the case $p=\infty$ we must show $r(\mu) \rightarrow 0$ as $\mu \rightarrow 0$ where $r(\mu)=\left\|\left[j^{n}(v, \lambda+\mu)-j^{n}(v, \lambda)\right] \mu^{-1}-j_{\lambda}^{n}(v, \lambda)\right\|_{\infty}$. Note that (2.4) implies that $j_{\lambda}^{n}(v, \lambda)$ is in $L^{\infty}(\Omega)$ for each $\lambda$. Since $j^{n}(v, \lambda+\mu)-$ 


$$
\begin{aligned}
j^{n}(v, \lambda)= & \int_{\lambda}^{\lambda+\mu} j_{\lambda}^{n}(v, \eta) d \eta \text { a computation shows that } \\
r(\mu) & \leqq \sup \left\{\left|j_{\lambda}^{n}(v(x), \lambda+t \mu)-j_{\lambda}^{n}(v(x), \lambda)\right|: x \in \Omega, 0 \leqq t \leqq 1\right\} \\
& \leqq|\mu| \sup \left\{\left|j_{\lambda \lambda}^{n}(v(x), \lambda+t \mu)\right|: x \in \Omega, 0 \leqq t \leqq 1\right\} .
\end{aligned}
$$

Using (2.5) we get $r(\mu) \leqq 4|\mu|\|v\|_{\infty}[\operatorname{dist}(\lambda, \partial S)]^{2}$ for $|\mu|<$ $2^{-1} \operatorname{dist}(\lambda, \partial S)$. Thus $r(\mu) \rightarrow 0$.

A special case of this example is $\beta(z)=z^{2}, z \in K \equiv\{z \in C:|\arg z| \leqq$ $\pi / 4\} \cup\{0\}$. We have $\left|\arg \beta^{\prime}(z)\right| \leqq \pi / 4, x \in K$, so we can take $\theta=\pi / 4$. Note that $(I+\lambda \beta)^{-1} w=\left[-1+(1+4 \lambda w)^{1 / 2}\right] / 2 \lambda$ for $w \in R(I+\lambda \beta)$, $|\arg \lambda|<\pi / 4$. A simple geometric argument shows that if $|\arg \lambda|<$ $\pi / 4$ and $|\arg w| \leqq \pi / 4(\operatorname{resp} .|\arg w|<\pi / 4)$ then $\left|\arg (I+\lambda \beta)^{-1} w\right| \leqq$ $\pi / 4$ (resp. $<\pi / 4)$. Thus $K \subset(I+\lambda \beta)(K)$ and $U \subset(I+\lambda \beta)(U)$, $|\arg \lambda|<\pi / 4$, where $U$ is the interior of $K$.

To obtain an "unbounded generator" version of the above example, let $X=l^{2}, D(A)=\left\{x \in l^{2}: A x \in l^{2},\left|\arg x_{i}\right| \leqq \pi / 4\right\}$. Let $\Sigma_{\theta}=$ $\{\lambda \in C:|\arg \lambda|<\pi / 4\}$ and let $A\left(x_{1}, x_{2}, x_{3}, \cdots\right)=\left(x_{1}^{2}, 2 x_{2}^{2}, 3 x_{3}^{2}, \cdots\right)$. The hypotheses of Theorem 1 are easy to verify in this case.

Our results include some, but not all, of the linear theory of holomorphic semigroups. If $A$ is an $m$-sectorial operator in a Hilbert space with vertex zero (so that its numerical range is a subset of a sector $|\arg \varphi| \leqq \pi / 2-\theta, \theta<\pi / 2)$, then $A$ satisfies the hypotheses of Theorem 2.1.

3. A perturbation theorem. In this section we consider the equation $d u / d t+T u(t)=F u(t), t \geqq 0, u(0)=x$, where $T$ is a linear operator in a complex Banach space $X$ and $F$ is a function with domain and range in $X$. Equations of this type have been studied by Sobolevskii [23], Fujita and Kato [9], Friedman [8], Henry [10] and others. We establish analyticity in $t$ of solutions $u(t)$ of this equation under suitable conditions on $T$ and $F$. In particular, we assume that

The resolvent of $T$ exists for $\operatorname{Re} \lambda \leqq 0$ and there exists

a constant $C$ such that $\left\|(\lambda-T)^{-1}\right\| \leqq C(1+|\lambda|)^{-1}$, $\operatorname{Re} \lambda \leqq 0$.

Using the Neumann series representation for the resolvent [12, pp. 37,173$]$ it is not hard to show that there exists $C_{1}, \omega>0$ such that the resolvent of $T$ exists and satisfies $\left\|(\lambda-T)^{-1}\right\| \leqq C_{1}|\lambda|^{-1}$ for $|\arg \lambda-\pi|<(\pi / 2)+\omega$. This is a well known ([12, p. 488], [8, p. 101]) condition for $-T$ to generate a holomorphic semigroup $\{U(t):|\arg t|<\omega\}$. The map $t \rightarrow U(t)$ is a bounded holomorphic map from $\{t:|\arg t|<\theta$, $t \neq 0\}$ into $B(X)$ for any $\theta<\omega$. 
The assumption (3.1) implies that $T$ has fractional powers, $T^{r}$, for $\gamma \in \boldsymbol{R}$ (see $[24,8,18]$ ). For $\gamma \leqq 0, T^{\gamma} \in B(X)$. For $\gamma \geqq 0, T^{\gamma}$ is a closed operator in $X$ with domain, $X_{r} \equiv D\left(T^{\gamma}\right)$, dense in $X$. For all $\gamma, T^{r}$ is invertible with $\left(T^{r}\right)^{-1}=T^{-r}$; see [8, pp. 158-159]. For $\gamma>0$, we define $\|x\|_{r}=\left\|T^{\gamma} x\right\|, x \in X_{\gamma}$ (cf. [10, p. 29]). The fact that $\left(T^{r}\right)^{-1} \in B(X)$ implies \|\|$_{r}$ is a norm on $X_{r}$ which is equivalent to the graph norm, II $\|$, of $T^{\gamma}$, since $\|x\| \equiv\left\|T^{\gamma} x\right\|+\|x\| \leqq\left(1+\left\|T^{-\gamma}\right\|\right)\left\|T^{\gamma} x\right\| \cdot X_{r}$ is a Banach space with the norm \|\|$_{r}$ since $T^{r}$ is a closed operator. In $\S 4$ we shall need the following imbedding theorem for domains of factional powers.

If $Y$ is a Banach space with $D(T) \subset Y \subset X$ and $0 \leqq \beta<1$ and there exists $C$ such that $\|u\|_{Y} \leqq C\|T u\|_{X}^{\beta}\|u\|_{X}^{1-\beta}$, $u \in D(T)$, then $D\left(T^{\alpha}\right)$ is continuously imbedded in $Y$ for $\beta<\alpha \leqq 1$.

(See Sobolevskii [23, p. 22], Friedman [8, p. 177], and Henry [10, p. 29].)

We shall also need the following facts which relate the semigroup to the fractional powers. For all $\gamma \geqq 0, U(T)$ maps $X$ into $D\left(T^{r}\right)$ and, for $\theta<\omega$ there exists a constant $M_{r}$ such that

$$
\left\|T^{\gamma} U(t)\right\| \leqq M_{\gamma}|t|^{-\gamma}, \quad|\arg t|<\theta .
$$

(See [8, pp. 105-106, 158-160] where this is proved for real $t$. The same argument works for complex $t$.)

For $0<\gamma \leqq 1, \theta<\omega$ one has

$$
\|U(t) x-x\| \leqq M_{1-\gamma} \gamma^{-1}|t|^{\gamma}\left\|T^{\gamma} x\right\|,
$$

$|\arg t|<\theta, x \in X_{r}$. (To prove this, note that $(d / d s) U(s) x=-T U(s) x=$ $-T^{1-\gamma} U(s) T^{\gamma} x$. Thus $U(t) x-x=-\int_{0}^{t} T^{1-r} U(s) T^{\gamma} x d s$. Using (3.3) to estimate $\left\|T^{1-r} U(s)\right\|$, one obtains (3.4). This proof is due to Henry [10].)

Let $1<p \leqq \infty, 0 \leqq \gamma<1-p^{-1}, 0<\varepsilon<\tau$. Then there exists a constant $M$ such that if $u:[0, \tau] \rightarrow X$ is differentiable, $u(t) \in D(T)$, $0 \leqq t \leqq \tau$, and $u^{\prime}(t)+T u(t)=f(t), 0 \leqq t \leqq \tau$, with $f \in L^{p}(0, \tau ; X)$ then

$$
\left\|T^{r} u(t)\right\| \leqq M\left[\|u(0)\|+\left(\int_{0}^{\tau}\|f(s)\|^{p} d s\right)^{1 / p}\right],
$$

$\varepsilon \leqq t \leqq \tau$. To prove (3.5), first note that

$$
u(t)=U(t) u(0)+\int_{0}^{t} U(t-s) f(s) d s
$$

(see [12, p. 486]). By (3.3) we have $\left\|T^{r} U(t) u(0)\right\| \leqq M_{\gamma} \varepsilon^{-r}\|u(0)\|$, 
$\varepsilon \leqq t \leqq \tau, \quad$ and $\quad \int_{0}^{t}\left\|T^{\gamma} U(t-s) f(s)\right\| d s \leqq M_{r} \int_{0}^{t}\|f(s)\|(t-s)^{-\gamma} d s \leqq$ $M_{r}\left(\int_{0}^{t}\|f(s)\|^{p} d s\right)^{1 / p}\left(\int_{0}^{t}(t-s)^{-\gamma^{q}}\right)^{1 / q} \leqq$ const. $\left(\int_{0}^{\tau}\|f(s)\|^{p} d s\right)^{1 / p}, 0 \leqq t \leqq \tau$, $p^{-1}+q^{-1}=1$. Note that $\gamma q<1$ since $\gamma<q^{-1}=1-p^{-1}$. This proves (3.5).

Theorem 3.1. Assume T satisfies (3.1), $0 \leqq \alpha<1, \theta<\omega$, and $F$ is a function whose domain, $D(F)$, is an open subset of $X_{\alpha}$ and $F: D(F) \rightarrow X$ is Frechet analytic (as a map from $X_{\alpha}$ to $X$ ). Then for each $x \in D(F)$ there exists $r>0$ and a unique function $u$ mapping $W_{r}=\{t \in C:|\arg t|<\theta, 0<|t|<r\}$ analytically into $X_{1}=D(T)$ such that for each $t \in W_{r}, u(t) \in D(F)$ and $u^{\prime}(t)+T u(t)=F u(t)$, and $\|u(t)-x\|_{\alpha} \rightarrow 0$ as $t \rightarrow 0$.

Let $U \subset D(F) \cap X_{r}$ for some $\gamma>\alpha$ and suppose there exists $\delta>0$ and $K$ such that if $x \in U$ and $\|y-x\|_{\alpha}<\delta$ then $y \in D(F)$ and $\|F y\|<K$. Suppose also that $U$ is bounded in $X_{r}$. Then the value of $r$ can be chosen independently of $x \in U$.

If, in addition, $F$ maps $D(F) \cap X_{s+\alpha}$ analytically into $X_{s}$ for $0 \leqq s \leqq n$, then $u$ is analytic from $W_{r}$ to $X_{n+1}$.

Proof. The differential equation $d u / d t+A u=F u$ is transformed into the integral equation (3.7) below. This method was introduced by Sobolevskii [23] and Fujita and Kato [9] and is now standard. We use methods similar to Henry [10], and therefore we are as brief as possible.

Choose $\delta>0$ and $K$ so that $\|y-x\|_{\alpha}<\delta$ implies $y \in D(F)$ and $\|F y\| \leqq K$. Using the Cauchy integral formula, one has

$$
\left\|F y_{1}-F y_{2}\right\| \leqq 4 K \delta^{-1}\left\|y_{1}-y_{2}\right\|_{\alpha},
$$

if $\left\|y_{i}-x\right\|_{\alpha} \leqq \delta / 2, i=1,2$. Let $S_{r}$ be the set of all analytic functions $u: W_{r} \rightarrow X_{\alpha}$ such that $\|u(t)-x\|_{\alpha} \leqq \delta / 2, t \in W_{r}$ and $\|u(t)-x\|_{\alpha} \rightarrow 0$ as $t \rightarrow 0 . S_{r}$ is a complete metric space if we define $d(u, v)=$ $\sup \times\left\{\|u(t)-v(t)\|_{\alpha}: t \in W_{r}\right\}, u, v \in S_{r}$.

For $u \in S_{r}$ put

$$
G u(t)=U(t) x+\int_{0}^{t} U(t-s) F u(s) d s, \quad t \in W_{r},
$$

where the integral is taken over the line segment $\{s=\lambda t, 0 \leqq \lambda \leqq 1\}$ joining 0 to $t$. We shall show $G$ is a strict contraction from $S_{r}$ into $S_{r}$ if $r$ is chosen small enough.

First consider the integral on the right of (3.7); we denote its value by $v(t)$. Putting $s=\lambda t, 0 \leqq \lambda \leqq 1$, we get $v(t)=t \int_{0}^{1} g(t, \lambda) d \lambda$ where $g(t, \lambda)=U(t-t \lambda) f(t \lambda)$, where $f(t)=F u(t)$. Using (3.3) one 
sees that there is a constant $C$ such that $\|g(t, \lambda)\|_{\alpha} \leqq C|t|^{-\alpha}(1-\lambda)^{-\alpha}$, $t \in W_{r}, 0<\lambda<1$. Thus the integral in (3.7) is absolutely convergent in $X_{\alpha}$ and $\|v(t)\|_{\alpha} \leqq C_{1}|t|^{1-\alpha}, t \in W_{r}$. In particular, $\|v(t)\|_{\alpha} \rightarrow 0$ as $t \rightarrow 0$, and we can make $\|v(t)\|_{\alpha} \leqq \delta / 4, t \in W_{r}$, by choosing $r$ sufficiently small.

Since $\|U(t) x-x\|_{\alpha}=\left\|U(t) T^{\alpha} x-T^{\alpha} x\right\|$ approaches 0 as $t \rightarrow 0$, we can make $\|U(t) x-x\|_{\alpha}<\delta / 4$ by making $r$ small. If $x \in X_{r}$ for some $\gamma>\alpha$, then the size of $r$ necessary to make $\|U(t) x-x\|_{\alpha}<\delta / 4$ is determined by $\|x\|_{r}$. This is because (3.4) implies

$$
\|U(t) x-x\|_{\alpha} \leqq \text { const. }|t|^{\gamma-\alpha}\left\|T^{\gamma-\alpha} T^{\alpha} x\right\| \leqq \text { const. }|t|^{\gamma-\alpha}\|x\|_{r} .
$$

Combining these results, one has $\|G u(t)-x\|_{\alpha} \rightarrow 0$ as $t \rightarrow 0$, and $\|G u(t)-x\|_{\alpha} \leqq \delta / 2, t \in W_{r}$ for $r$ small.

Since $U(t) x$ is analytic in $t$, it remains to show the integral $v(t)$ is analytic in $t$ with values in $X_{\alpha}$. For fixed $\lambda \in(0,1), g(t, \lambda)$ is an analytic function of $t$ with values in $X_{\alpha}$ and

$$
g_{t}(t, \lambda)=-(1-\lambda) T U(t-t \lambda) f(t \lambda)+U(t-t \lambda) f^{\prime}(t \lambda) \lambda,
$$

where $g_{t}=\partial g / \partial t$. The function $f$ is bounded by $K$, so by the Cauchy integral formula $\left\|f^{\prime}(t)\right\| \leqq K|t|^{-1} \csc (\theta-|\arg t|)$. Using this and (3.3), one sees that $\left\|g_{t}(t, \lambda)\right\|_{\alpha}$ is bounded by const. $(1-\lambda)^{-\alpha}$ for $t$ in a compact subset of $W_{r}$. Thus the difference quotients $\|[g(t, \lambda)-$ $g(s, \lambda)] /(t-s) \|_{\alpha}$ are similarly bounded. Using the dominated convergence theorem, it follows that $v: W_{r} \rightarrow X_{\alpha}$ is analytic. Therefore $G u: W_{r} \rightarrow X_{\alpha}$ is analytic.

We have shown $G$ maps $S_{r}$ into $S_{r}$ for $r$ small. To show $G$ is a contraction, we use (3.3) and (3.6) to get

$$
\begin{aligned}
\|G u(t)-G v(t)\|_{\alpha} & \leqq M_{\alpha} \int_{0}^{t}|t-s|^{-\alpha}\|F u(s)-F u(s)\| d|s| \\
& \leqq \text { const. }|t|^{1-\alpha} \sup \|u(s)-v(s)\|_{\alpha},
\end{aligned}
$$

$t \in W_{r}, u, v \in S_{r}$. By making $r$ sufficiently small we can make $G$ a strict contraction. By the fixed point theorem for strict constractions on a complete metric space, there is a unique $u \in S_{r}$ such that $G u=u$. In order to show $u$ satisfies the differential equation $u^{\prime}(t)+T u(t)=F u(t)$ we will use a known result (see Kato [12], Theorem 1.27, p. 491) on solutions to inhomogeneous equations for holomorphic semigroups. In order to apply this theorem it is necessary to make two changes of variable. Fix $t \in W_{r}$ and define $v(\lambda)=u(\lambda t)=U(\lambda t) x+\int_{0}^{\lambda t} U(\lambda t-s) \times$ $F u(s) d s$. Putting $s=\sigma t, 0 \leqq \sigma \leqq \lambda$, we get $v(\lambda)=V(\lambda) x+\int_{0}^{\lambda} V(\lambda-\sigma) f(\sigma) d \sigma$ where $V(\lambda)=U(\lambda t)$ is the (holomorphic) semigroup generated by $-t T$, and $f(\sigma)=t F u(\sigma t)$ is continuous on $[0, r /|t|)$ and analytic on $(0, r /|t|)$ with values in $X$. Fixing $\tau<1$, it is not hard to show $v(\lambda+\tau)=$ 
$V(\lambda) v(\tau)+\int_{0}^{\lambda} V(\lambda-\rho) f(\rho+\tau) d \rho, 0 \leqq \lambda<r /|t|-\tau$. The function $\rho \mapsto f(\rho+\tau)$ is Hölder continuous on $[0, r /|t|-\tau)$. By the above mentioned theorem in [12], it follows that $v(s) \in D(T), \tau<s<r /|t|$, with $v^{\prime}(s)+t T v(s)=f(s)$. Putting $s=1$ shows $u(t) \in D(T)$ and $u^{\prime}(t)+T u(t)=F u(t)$. So far we know $u: W_{r} \rightarrow X_{\alpha}$ is analytic. If we rewrite the equation as $u=T^{-1}\left(F u-u^{\prime}\right)$ it follows that $u: W_{r} \rightarrow X_{1}$ is analytic. The solution of $u^{\prime}+T u=F u, u(0)=x$ is unique because any $u$ satisfying the conclusions of the theorem must also satisfy $G u=u$.

Suppose $F$ is analytic from $U \cap X_{s+\alpha}$ to $X_{s}, 0 \leqq s \leqq n$. If $u$ is analy tic from $W_{r}$ to $X_{s+\alpha}$ for such an $s$, then the equation $u=T^{-1}\left(F u-u^{\prime}\right)$ shows $u: W_{r} \rightarrow X_{s+1}$ is analytic. Repeating this argument shows that $u: W_{r} \rightarrow X_{n+1}$ is analytic.

4. Semilinear parabolic equations. In this section the results of $\S 3$ are applied to the mixed problem $\partial u / \partial t+L u+\beta(u)=0$, $(x, t) \in \Omega \times[0, \infty) ; u(x, 0)=\varphi(x), x \in \Omega ; u(x, t)=0,(x, t) \in \partial \Omega \times[0, \infty) ;$ where $L$ is a second order elliptic operator of the form $L u=$ $-\sum_{i, j} \partial_{j}\left[a_{i j} \partial_{i} u\right]+\sum_{i} \partial_{i}\left[a_{i} u\right]+a u$. Here $\partial_{j}=\partial / \partial x_{j}$ and sums are from 1 to $n . \quad \Omega$ is the closure of a bounded, open subset of $\boldsymbol{R}^{n}$, and $\Omega$ has smooth boundary $\partial \Omega$. The $a_{i j}, a_{i}, a$ are real valued functions on $\Omega$ with $a_{i j}=a_{j i} ; a_{i j}, a_{i} \in C^{1}(\Omega), a \in C(\Omega)$ and there exists $\mu>0$ such that $\sum_{i j} a_{i j} \xi_{i} \xi_{j} \geqq \mu|\xi|^{2}, \xi \in \boldsymbol{R}^{n}, x \in \Omega$. $\quad \beta$ is an analytic function whose domain, $D(\beta)$, is an open subset of the complex plane containing the real axis; $\beta$ maps the real line into itself; for $t$ real, $\beta(t)$ is an increasing function of $t$, and $\beta(0)=0$.

Equations of this type have been studied by Brezis, Crandall and Pazy [3], Brezis and Strauss [4], Da Prato [7], Konishi [17], Ouchi [22], and Brezis [2]. Our main result is that the solution of the mixed problem above is an analy tic function of $t>0$; see Theorem 4.4 below. This result is similar to those of Ouchi, but he only considers the case where $\beta$ is a polynomial.

$W^{k, p}(\Omega ; \boldsymbol{R})$ (resp. $W^{k, p}(\Omega ; \boldsymbol{C})$ ) is the Sobolev space of real-valued (resp. complex-valued) functions whose derivatives up to order $k$ lie in $L^{p}(\Omega ; \boldsymbol{R})\left(\operatorname{resp} . L^{p}(\Omega ; \boldsymbol{C})\right)$. We write $W^{k, p}(\Omega)$ if it is clear from the context whether $\boldsymbol{R}$ or $\boldsymbol{C}$ is intended. The norm in $W^{k, p}(\Omega)\left(\operatorname{resp} . L^{p}(\Omega)\right)$ is denoted by \|\|$_{k, p}$ (resp. \|\|$\left._{p}\right) . \quad W_{0}^{k, p}(\Omega)$ is the closure of $C_{0}^{\infty}\left(\Omega^{0}\right)$ in the space $W^{k, p}(\Omega)$. Here $\Omega^{0}$ is the interior of $\Omega$. If $u$ is a function, then $\beta(u)=\beta \circ u$ is the composition of $\beta$ and $u$.

For $1<p<\infty$, let $D\left(T_{p}\right)=W^{2, p}(\Omega ; C) \cap W_{0}^{1, p}(\Omega)$ and, for $p=1$, let $D\left(T_{1}\right)=\left\{u \in W^{1,1}(\Omega ; C): L u \in L^{1}(\Omega)\right\}$, where $L u$ is understood in the sense of distributions. Let $T_{p} u=L u$ for $u \in D\left(T_{p}\right), 1 \leqq p<\infty$, For $1 \leqq p<\infty$, let $D\left(A_{p}\right)=\left\{u \in L^{p}(\Omega ; R): u \in D\left(T_{p}\right), \beta(u) \in L^{p}(\Omega)\right\}$, and $A_{p} u=T_{p} u+\beta(u), u \in D\left(A_{p}\right)$. 
Proposition 4.1. If $1<p<\infty$ and $k \in \boldsymbol{R}$ is sufficiently large, then $X=L^{p}(\Omega, C)$ and $T=T_{p}+k I$ satisfy (3.1) and there exists $a$ constant $C_{p}$ such that $\|u\|_{2, p} \leqq C_{p}\|T u\|_{p}, u \in D(T)$. If $0<\alpha \leqq 1$ and $p^{-1}-2 \alpha n^{-1}<q^{-1}$ then $X_{\alpha} \equiv D\left(T^{\alpha}\right)$ is continuously imbedded in $L^{q}(\Omega)$ (or $C(\Omega)$ if $q=\infty ; q=\infty$ corresponds to $n / 2 p<\alpha \leqq 1$ ).

Let $D(F)=\left\{u \in X_{\alpha}: u(x) \in D(\beta), x \in \Omega\right\}$ and $F u=k u-\beta(u), u \in D(F)$. If $n / 2 p<\alpha<1$ then $D(F) \subset C(\Omega)$ and $\beta(u) \in C(\Omega)$ for each $u \in D(F)$. Furthermore $X, T, \alpha$ and $F$ satisfy the hypotheses of Theorem 3.1. Let $R>0$ and $\triangle$ be a compact subset of $D(\beta)$ and $U=\{u \epsilon$ $\left.W^{2, p}(\Omega ; C):\|u\|_{2, p}<R ; u(x) \in \Delta, x \in \Omega\right\}$. Then $U$ also satisfies the hypotheses of Theorem 3.1.

Proof. The assertions in the first sentence are well known, see Sobolevskii [23, p. 54] and Friedman [8, p. 101]. If $p^{-1}-2 \alpha n^{-1}<q^{-1}$ then it follows from Friedman [8, Theorems 10.1, 11.1] that $W^{2, p}(\Omega) \subset$ $L^{q}(\Omega)$ (or $W^{2, p}(\Omega) \subset C(\Omega)$ if $q=\infty$ ) and there is $\mu<\alpha$ and $C$ such that $\|u\|_{q} \leqq C\|u\|_{2, p}^{\mu}\|u\|_{p}^{1-\mu}, u \in W^{2, p}(\Omega)$. Thus $\|u\|_{q} \leqq C\|T u\|_{p}^{\mu}\|u\|_{p}^{1-\mu}$, $u \in D(T)$. Thus $X_{\alpha} \subset L^{q}(\Omega)$ follows from (3.2).

Now let $n / 2 p<\alpha<1$. The fact that $D(F) \subset C(\Omega)$ follows from the first part of the proposition, and $\beta(u) \in C(\Omega), u \in D(F)$ follows from the fact that $\beta$ is continuous. To show that $D(F)$ is open in $X_{\alpha}$, let $u \in D(F)$. Then $u(\Omega) \equiv\{u(x): x \in \Omega\}$ is compact and contained in $D(\beta)$ which is open. Thus, the distance, $\delta$, from $u(\Omega)$ to $C \backslash D(\beta)$ is greater than 0 . It follows that $v(x) \in D(\beta)$ if $\|v-u\|_{\infty}<\delta$. Since $X_{\alpha} \subset C(\Omega)$ one has $\|v-u\|_{\infty}<\delta$ if the $X_{\alpha}$ norm of $v-u$ is sufficiently small. Thus $D(F)$ is open in $X_{\alpha}$.

To show $F: D(F) \rightarrow X$ is analytic, it sufficies to show $\| F(u+h)-$ $F(u)-\left(k h-\beta^{\prime}(u) h\right)\left\|_{p} \leqq \varepsilon(h)\right\| T^{\alpha} h \|$ where $\varepsilon(h) \rightarrow 0$ as $\left\|T^{\alpha} h\right\| \rightarrow 0$. In view of the imbeddings $X_{\alpha} \subset C(\Omega) \subset X$, it suffices to show $\| \beta(u+h)-$ $\beta(u)-\beta^{\prime}(u) h\left\|_{\infty} \leqq \varepsilon(h)\right\| h \|_{\infty}$ where $\varepsilon(h) \rightarrow 0$ as $\|h\|_{\infty} \rightarrow 0$. By writing $\beta(\eta+\xi)-\beta(\eta)$ as the integral of $\beta^{\prime}$, one can show $\mid \beta(\eta+\xi)-\beta(\eta)-$ $\beta^{\prime}(\eta) \xi|\leqq \varepsilon(|\xi|)| \xi \mid, \eta \in u(\Omega)$, where $\varepsilon(|\xi|) \rightarrow 0$ as $|\xi| \rightarrow 0$ and $\varepsilon(|\xi|)$ is independent of $\eta \in u(\Omega)$. Replacing $\eta$ by $u(x)$ and $\xi$ by $h(x)$ and taking the supremum over $\Omega$, one obtains the desired result.

Note that $U$ is a bounded subset of $D(T)=X_{1}$. Since $\Delta \subset D(\beta)$ is compact, there exists $\rho>0$ such that $\Delta_{1}=\{z+\zeta: z \in \Delta,|\zeta| \leqq \rho\} \subset D(\beta)$. Using an argument similar to the proof that $D(F)$ is open in $X_{\alpha}$, one can find a $\delta>0$ such that if $u \in U$ and the $X_{\alpha}$ norm of $v-u$ is less than $\delta$ then $v(x) \in \Delta_{1}, x \in \Omega$, and hence, $v \in D\left(F^{\prime}\right)$. One has $\|F v\| \leqq K$ since $\beta$ is bounded on $A_{1}$.

Proposition 4.2. If $k \in \boldsymbol{R}$ is sufficiently large, then $\left(I+\lambda\left(A_{p}+k\right)\right)^{-1}$ exists and is a contraction in the norm of $L^{p}(\Omega)$ and the range of $I+\lambda\left(A_{p}+k\right) \quad$ is $L^{p}(\Omega ; \boldsymbol{R})$ for $1 \leqq p<\infty, \lambda>0$. Furthermore 
$\|\beta(u)\|_{p} \leqq\left\|\left(A_{p}+k\right) u\right\|_{p},\left\|\left(T_{p}+k\right) u\right\| \leqq 2\left\|\left(A_{p}+k\right) u\right\|_{p}, u \in D\left(A_{p}\right) . \quad$ If $\gamma: \boldsymbol{R} \rightarrow \boldsymbol{R}$ is increasing and continuous with $\gamma(0)=0, p^{-1}+q^{-1}=1$, $u \in D\left(T_{p}\right) \cap L^{p}(\Omega ; \boldsymbol{R})$ and $\gamma(u) \in L^{q}(\Omega)$ then $\int_{\Omega}\left(T_{p} u+k u\right) \gamma(u) d x \geqq 0$.

Proof. Most of the assertions follow from the results of Brezis and Strauss [4], so we are quite brief and only indicate how to apply their results. Let $k$ be such that $a(x)+k \geqq 0$ and $a(x)+\sum_{j} \partial_{j} \alpha_{j}(x)+$ $k \geqq 0, x \in \Omega$. Then the operator $L+k$ satisfies the hypotheses of Theorem 8 of [4]. Thus $T_{1}+k$ (when restricted to $D\left(T_{1}\right) \cap L^{1}(\Omega ; R)$ ) satisfies Proposition 7 of [4], and Lemma $3^{*}$ of [4] can be applied to $\left(I+\lambda\left(T_{1}+k\right)\right)^{-1}$. It follows that the range of $I+\lambda\left(A_{1}+k\right)$ is $L^{1}(\Omega ; \boldsymbol{R}),\left(I+\lambda\left(A_{1}+k\right)\right)^{-1}$ exists and it is a contraction with respect to any norm \|\|$_{p}, 1 \leqq p<\infty$. In particular, $\left(I+\lambda\left(A_{1}+k\right)\right)^{-1}$ maps $L^{p}(\Omega ; R)$ into $D\left(A_{1}\right) \cap L^{p}(\Omega)$. Since $A_{1}$ is an extension of $A_{p},(I+$ $\left.\lambda\left(A_{p}+k\right)\right)^{-1}$ exists and is a contraction in the norm \|\|$_{p}, 1 \leqq p<\infty$. We still need to show that the range of $I+\lambda\left(A_{p}+k\right)$ is $L^{p}(\Omega)$. Note that the linear operator $\lambda\left(T_{1}+k\right)$ and the monotone function $u \rightarrow$ $u+\lambda \beta(u)$ satisfy the hypotheses of Theorem 1 of [4]. Let $f \in L^{p}(\Omega ; \boldsymbol{R})$ and $u=\left(I+\lambda\left(A_{1}+k\right)\right)^{-1} f$. As noted above Lemma $3^{*}$ of [4] implies $u \in L^{p}(\Omega) \cap D\left(A_{1}\right)$, and Proposition 4 of [4] implies $u+\lambda \beta(u) \in L^{p}(\Omega)$, and, hence, $\beta(u)$ and $T_{1} u$ belong to $L^{p}(\Omega)$. Using regularity theorems [1] for linear elliptic operators we conclude $u \in W^{2, p}(\Omega)$, and, hence, $u \in D\left(A_{p}\right)$. Thus, the range of $I+\lambda\left(A_{p}+k\right)$ is $L^{p}(\Omega)$.

To prove the last part of the proposition, note that $T_{1}+k$ satisfies the hypotheses of Theorem 1 of [4]. Let $u \in D\left(A_{p}\right)$ and $f=\left(A_{p}+k\right) u$. By Proposition 4 of [4] we have $\|\beta(u)\|_{p} \leqq\left\|\left(A_{p}+k\right) u\right\|_{p}$ and, hence, $\left\|\left(T_{p}+k\right) u\right\|_{p} \leqq 2\left\|\left(A_{p}+k\right) u\right\|_{p}$. Using Lemma 2 of [4] we get $\int_{\Omega}\left(T_{p} u+k u\right) \gamma(u) d x \geqq 0$.

Proposition 4.3. Let $k$ be such that Propositions 4.1 and 4.2 are true.

(1) If $\varphi \in L^{1}(\Omega ; R)$ then $\lim _{(n \rightarrow \infty)}\left(I+(t / n) A_{1}\right)^{-n} \varphi \equiv u(t) \equiv S(t) \varphi$ exists in $L^{1}(\Omega)$ for all $t \geqq 0$. If $\varphi \in L^{p}(\Omega ; \boldsymbol{R})$ for some $p, 1 \leqq p<\infty$, then this limit exists in $L^{p}(\Omega), u:[0, \infty) \rightarrow L^{p}(\Omega)$ is continuous and $S(t): L^{p}(\Omega) \rightarrow L^{p}(\Omega)$ is Lipschitz with constant $e^{k t}$. In particular, $\|u(t)\|_{p} \leqq e^{k t}\|\varphi\|_{p}$.

(2) If $1<p<\infty$ and $\varphi \in D\left(A_{p}\right)$ then $u(t) \in D\left(A_{p}\right), t \geqq 0, u:[0, \infty) \rightarrow$ $L^{p}(\Omega)$ is absolutely continuous, the right derivative, $D_{r} u(t)$ exists and is equal to $-A_{p} u(t)$ for all $t \geqq 0$, and $\left\|A_{p} u(t)\right\|_{p} \leqq e^{k t}\left\|A_{p} \varphi\right\|_{p}$.

(3) If $n / 2 p<\alpha<1$ and $u\left(t_{0}\right) \in D\left(\left(T_{p}+k\right)^{\alpha}\right) \cap L^{p}(\Omega ; R)$ for some $t_{0} \geqq 0$, then $u:\left(t_{0}, \infty\right) \rightarrow W^{2, p}(\Omega) \cap W_{0}^{1, p}(\Omega)$ is analytic.

Proof. The first part of Proposition 4.2 says that $A_{p}+k I$ is 
$m$-accretive as defined by Kato $[14$, p. 138]. The assertions in part (1) are a direct application of the results of Crandall and Liggett [5, Theorem 1]. The fact that $\beta(0)=0$ implies $A_{1} \psi=0$ for $\psi=0$. Thus $S(t) \psi=0$ if $\psi=0$. This fact combined with the fact that $S(t)$ has Lipschitz constant $e^{k t}$ proves $\|u(t)\|_{p} \leqq e^{k t}\|\varphi\|_{p}$.

If $1<p<\infty$ then $L^{p}(\Omega)$ and its dual are uniformly convex and, if $\varphi \in D\left(A_{p}\right)$, the results of Kato [14, Theorems 7.1, 7.5 and first line of last paragraph of p. 147] imply $u$ has the properties in (2). (Note that the solution constructed by Kato in [14, Theorem 7.1, 7.5] coincides with $u(t)$ by virtue of [5, Theorem 2].)

To prove (3), let $n / 2 p<\alpha<1$ and $u\left(t_{0}\right) \in D\left(\left(T_{p}+k\right)^{\alpha}\right) \cap L^{p}(\Omega ; \boldsymbol{R})$. By Proposition 4.1 and Theorem 3.1 there exists $r>0$ and a continuous function $v:\left[t_{0}, t_{0}+r\right) \rightarrow L^{p}(\Omega)$ such that $v:\left(t_{0}, t_{0}+r\right) \rightarrow W^{2, p}(\Omega)$ is analytic, $v_{t}+\left(T_{p}+k\right) v=k v-\beta(v), t_{0}<t<t_{0}+r$, and $v\left(t_{0}\right)=u\left(t_{0}\right)$. Since $v$ satisfies Definition 2.2 of [5] for being a strong solution of $v_{t}+A_{p} v=0, v\left(t_{0}\right)=u\left(t_{0}\right)$, it follows from Theorem 2 of [5] that $v=u$ on $\left[t_{0}, t_{0}+r\right)$. In particular, $u(t) \in D\left(A_{p}\right)$ for $t_{0}<t<t_{0}+r$. By part (2), $u(t) \in D\left(A_{p}\right), t_{0}<t<\infty$, and $\left\|A_{p} u(t)\right\|_{p}$ is bounded for $t$ in any interval of the form $t_{1} \leqq t \leqq t_{2}$ where $t_{0}<t_{1}<t_{2}<\infty$. By Propositions 4.1 and 4.2, $\left\|T_{p} u(t)\right\|_{p},\|u(t)\|_{2, p}$ and $\|u(t)\|_{\infty}$ are also bounded for $t_{1} \leqq t \leqq t_{2}$. Therefore $\Delta=\left\{u(t)(x): x \in \Omega, t_{1} \leqq t \leqq t_{2}\right\}$ is a bounded subset of $\boldsymbol{R}$. Again using Proposition 4.1 and Theorem 3.1, one sees that there exists $r>0$ such that for any $t_{3} \in\left[t_{1}, t_{2}\right]$ there is a continuous function $v:\left[t_{3}, t_{3}+r\right) \rightarrow L^{p}(\Omega)$ such that $v:\left(t_{3}, t_{3}+r\right) \rightarrow$ $W^{2, p}(\Omega)$ is analytic $v_{t}+A_{p} v(t)=0, t_{3}<t<t_{3}+r$, and $v\left(t_{3}\right)=u\left(t_{3}\right)$. As above, it follows from Theorem 2 of [5] that $u=v$ on $\left[t_{3}, t_{3}+r\right)$. Since $r$ is independent of $t_{3} \in\left[t_{1}, t_{2}\right]$, it follows that $u:\left(t_{1}, t_{2}\right) \rightarrow W^{2, p}(\Omega)$ is analytic. Since $t_{1}, t_{2}$ are arbitrary, it follows that $u:\left(t_{0}, \infty\right) \rightarrow W^{2, p}(\Omega)$ is analytic.

THEOREM 4.4. Let $\varphi \in W^{2, p}(\Omega ; \boldsymbol{R}) \cap W_{0}^{1, p}(\Omega)$ and $\beta(\varphi) \in L^{p}(\Omega)$, i.e. $\varphi \in D\left(A_{p}\right)$, for some $p, 1<p<\infty$. Then there exists a differentiable function $u:[0, \infty) \rightarrow L^{p}(\Omega ; \boldsymbol{R})$ such that $u:(0, \infty) \rightarrow W^{2, q}(\Omega ; \boldsymbol{R}) \cap W_{0}^{1, q}(\Omega)$ is analytic for all $q, 1 \leqq q<\infty, u_{t}+L u+\beta(u)=0,0 \leqq t<\infty$, and $u(0)=\varphi$. In fact $u(t)=S(t) \phi$ is constructed from $\varphi$ by Proposition 4.3 .

The proof of this theorem uses the a priori inequality in the following lemma. The authors wish to thank Professor H. Brezis for many helpful suggestions regarding this inequality.

Lemma 4.5. Let $k$ be such that Propositions 4.1 and 4.2 are true. Let $1<p \leqq q<\infty, 0 \leqq \alpha<1-q^{-1}, 0<\varepsilon<\tau$. Then there is an increasing function $l:(0, \infty) \rightarrow(0, \infty)$ such that if $\varphi \in W^{2, r}(\Omega: R) \cap$ 
$W_{0}^{1, r}(\Omega)=D\left(T_{r}\right)=D\left(A_{r}\right)$ for some $r \geqq q, r>n / 2$ then $\left\|\left(T_{q}+k\right)^{\alpha} u(t)\right\|_{q} \leqq$ $l\left(\left\|A_{p} \varphi\right\|_{p}+\|\varphi\|_{p}\right), \varepsilon \leqq t \leqq \tau$, where $u(t)=S(t) \varphi$ is obtained from $\varphi$ by Proposition 4.3.

Proof of Lemma 4.5. It follows from Proposition 4.3 that $u:(0, \infty) \rightarrow W^{2, r}(\Omega) \cap W_{0}^{1, r}(\Omega)$ is analytic, $u:[0, \infty) \rightarrow L^{r}(\Omega)$ is differentiable, $\left\|A_{r} u(t)\right\|_{r}$ is bounded for $t$ lying in any bounded interval and $u_{t}+\left(T_{r}+k\right) u=k u-\beta(u)$ holds for all $t \geqq 0$. From Proposition 4.1 and 4.2 it follows that $\|\beta(u(t))\|_{r},\left\|T_{r} u(t)\right\|_{r}$ and $\|u(t)\|_{2, r}$, are bounded for $t$ lying in any bounded interval. According to Proposition 4.1, the map $u \rightarrow \beta(u)$ is analytic from (an open subset of) $W^{2, r}(\Omega ; C)$ to $L^{r}(\Omega)$. Thus $t \rightarrow \beta(u(t))$ is an analytic function from $(0, \infty)$ to $L^{r}(\Omega)$ and bounded for $t$ lying in any bounded interval.

For $1<\rho \leqq r$ we may apply inequality (3.5) with $X=L^{\rho}(\Omega)$ and $T=T_{\rho}+k$ to obtain

$$
\left\|\left(T_{\rho}+k\right)^{\mu} u(t)\right\|_{\rho} \leqq C\left[\|u(\sigma)\|_{\rho}+\left(\int_{\sigma}^{\tau}\|k u-\beta(u)\|_{\rho}^{\rho} d t\right)^{1 / \rho}\right],
$$

$\sigma+\varepsilon / 2 \leqq t \leqq \tau, 0 \leqq \mu<1-\rho^{-1}$. Using Minkowski's inequality on the integral and estimating $\|u(t)\|_{\rho}$ in terms of $\|u(\sigma)\|_{\rho}$ (by Proposition 4.3) one obtains

$$
\left\|\left(T_{\rho}+k\right)^{\mu} u(t)\right\|_{\rho} \leqq C\left[\|u(\sigma)\|_{\rho}+\left(\int_{\sigma}^{\tau}\|\beta(u)\|_{\rho}^{\rho} d t\right)^{1 / \rho}\right],
$$

$\sigma+\varepsilon / 2 \leqq t \leqq \tau, 0 \leqq \mu<1-\rho^{-1}$. Applying Proposition 4.1 to the left side, one obtains

$$
\|u(t)\|_{s} \leqq C\left[\|u(\sigma)\|_{\rho}+\left(\int_{\sigma}^{\tau}\|\beta(u)\|_{\rho}^{\rho} d t\right)^{1 / \rho}\right],
$$

$\sigma+\varepsilon / 2 \leqq t \leqq \tau, \rho^{-1} \geqq s^{-1}>\rho^{-1}-2 \mu n^{-1}>\rho^{-1}-2 n^{-1}\left(1-\rho^{-1}\right)$. This is equivalent to $\rho \leqq s<\rho\left[1-2 n^{-1}(\rho-1)\right]^{-1}$ if $1-2 n^{-1}(\rho-1) \geqq 0$, and to $\rho \leqq s \leqq \infty$ if $1-2 n^{-1}(\rho-1)<0$.

We now show that there is an increasing function $l:(0, \infty) \rightarrow(0, \infty)$ such that

$$
\|u(t)\|_{q}+\int_{\sigma+\varepsilon}^{\tau}\|\beta(u)\|_{q}^{q} d t \leqq l\left(\|u(\sigma)\|_{p}+\int_{\sigma}^{\tau}\|\beta(u)\|_{p}^{p} d t\right),
$$

$\sigma+\varepsilon \leqq t \leqq \tau$. Let $\gamma(\xi)=|\beta(\xi)|^{q-2} \beta(\xi), \xi \in \boldsymbol{R}$. Multiplying the equation $\beta(u)=-u_{t}-\left(T_{q}+k\right) u+k u$ by $\gamma(u)$, integrating over $\Omega$, and using Proposition $4.2 \quad k u \gamma(u) \leqq C|u|^{q}+2^{-1}|\beta(u)|^{q}$, one obtains $\|\beta(u)\|_{q}^{q} \leqq-2 \int u_{t} \gamma(u) d x+C\|u\|_{q}^{q}, 0 \leqq t<\infty$. Let $\zeta: R \rightarrow R$ be smooth, $0 \leqq \zeta \leqq 1, \zeta=0$ on $(-\infty, \sigma+\varepsilon / 2]$, and $\zeta=1$ on $[\sigma+\varepsilon, \infty)$. Multiplying the above inequality by $\zeta$ and integrating from $\sigma$ to $\tau$, one obtains 


$$
\int_{\sigma+\varepsilon}^{\tau}\|\beta(u)\|_{q}^{q} d t \leqq-2 \int_{\sigma}^{\tau} \zeta(t) \int u_{t} \gamma(u) d x d t+C \int_{\sigma+\varepsilon / 2}^{\tau}\|u\|_{q}^{q} d t
$$

Let $\Gamma(\eta)=\int_{0}^{\eta} \gamma(\xi) d \xi, \eta \in R$. Then $\Gamma^{\prime}=\gamma, \Gamma(0)=0, \Gamma \geqq 0$. Since $\Gamma$ is convex, we have $\Gamma(0)-\Gamma(\eta) \geqq \gamma(\eta)(0-\eta)$ i.e. $\Gamma(\eta) \leqq \gamma(\eta) \eta$. Using the same argument that was used in the proof of Proposition 4.1, one can show that the map $G: u \rightarrow \Gamma(u)$ is Fréchet differentiable from $W^{2, r}(\Omega ; \boldsymbol{R})$ to $L^{r}(\Omega)$, and its differential is given by $D G(u) v=\gamma(u) v$. Therefore the map $t \rightarrow \Gamma(u(t))$ is differentiable from $(0, \infty)$ to $L^{r}(\Omega)$ and its derivative is $\gamma(u(t)) u_{t}(t)$. Thus $\int \gamma(u) u_{t} d x=(d / d t) \int \Gamma(u) d x$. If we integrate the first term on the right of (4.4) by parts, we get $\int_{\sigma}^{\tau} \zeta^{\prime}(t) \int \Gamma(u) d x d t-\int \Gamma(u(\tau)) d x($ since $\zeta(\tau)=1, \zeta(\sigma)=0)$. Using the fact that $\Gamma \geqq 0$ and $\Gamma(\eta) \leqq|\beta(\eta)|^{q-2} \beta(\eta) \eta$, one sees that the preceding integrals are dominated by $C \int_{o+\varepsilon / 2}^{\tau} \int|\beta(u)|^{q-1}|u| d x d t$. Applying Hölders inequality, one sees that this integral is dominated by $C \int_{\sigma+\varepsilon / 2}^{\tau}\|\beta(u)\|_{(q-1) a}^{q-1} \mid u \|_{b} d t$, where $a^{-1}+b^{-1}=1$. Using $x y \leqq a^{-1} x^{a}+$ $b^{-1} y^{b}$, one sees that this is dominated by $C \int_{o+\varepsilon / 2}^{\tau}\|\beta(u)\|_{(q-1) a}^{(q-1) a} d t+$ $C \int_{\sigma+\varepsilon / 2}^{\tau}\|u\|_{b}^{b} d t$. Let $p$ be fixed and choose $b$ so that (4.2) holds with $\rho$ replaced by $p$, i.e. $0 \leqq p^{-1}-b^{-1}<\min \left\{2 n^{-1}\left(1-p^{-1}\right), p^{-1}\right\}$. Then choose $q$ so that $(q-1) a=p$, i.e. $q=p\left(1+p^{-1}-b^{-1}\right)$. This implies $p \leqq q<\min \left\{p+1, p+2 n^{-1}(p-1)\right\}$. Then the integrals above are dominated by $l\left(\|u(\sigma)\|_{p}+\int_{\sigma}^{\tau}\|\beta(u)\|_{p}^{p} d t\right)$ where $l:(0, \infty) \rightarrow(0, \infty)$ is increasing. Putting this together with (4.4) gives

$$
\int_{\sigma+\varepsilon}^{\tau}\|\beta(u)\|_{q}^{q} d t \leqq l\left(\|u(\sigma)\|_{p}+\int_{\sigma}^{\tau}\|\beta(u)\|_{p}^{p} d t\right)+C \int_{\sigma+\varepsilon / 2}^{\tau}\|u\|_{q}^{q} d t .
$$

We restrict $q$ so that (4.2) holds with $s$ replaced by $q$ and $\rho$ replaced by $p$. Then the second term on the right of (4.5) can be estimated by the first term and we obtain the desired inequality (4.3) for $p \leqq q<\min \left\{p+1, p+2 n^{-1}(p-1), p\left[1+2 n^{-1}(p-1)\right]^{-1}\right\}$. However, we may now proceed to argue inductively on $p$ and $q$ to obtain (4.3) for all $p, q, 1<p \leqq q<\infty$.

To finish the proof of the lemma, note that Proposition 4.3 implies $\left\|\left(A_{p}+k\right) u(t)\right\|_{p} \leqq C\left(\left\|A_{p} \varphi\right\|_{p}+\|\varphi\|_{p}\right), 0 \leqq t \leqq \tau$. Combining this with Proposition 4.2, one obtains $\|\varphi\|_{p}+\int_{0}^{\tau}\|\beta(u)\|_{p}^{p} d t \leqq l\left(\left\|A_{p} \varphi\right\|_{p}+\|\varphi\|_{p}\right)$. Combining this with (4.3), one obtains $\|u(t)\|_{q}+\left(\int_{\varepsilon / 2}^{\tau}\|\beta(u)\|_{q}^{q} d t\right)^{1 / q} \leqq$ $l\left(\left\|A_{p} \varphi\right\|_{p}+\|\varphi\|_{p}\right), \varepsilon / 2 \leqq t \leqq \tau$. Using (4.1) with $\rho$ replaced by $q$ and $\mu$ replaced by $\alpha$, one obtains the inequality in the lemma.

Proof of Theorem 4.4 Since $\Omega$ is bounded it suffices to prove the theorem for all $q$ sufficiently large. We choose $q$ so large than 
$n / 2 q<\alpha<1-q^{-1}$, and then pick $\alpha$ so that $n / 2 q<\alpha<1-q^{-1}$. For such $q$ and $\alpha$ we can apply Proposition 4.3 (part (3)) and Lemma 4.5.

There exists a sequence $\left\{\varphi_{n}\right\} \subset W^{2, q}(\Omega ; \boldsymbol{R}) \cap W_{0}^{1, q}(\Omega)$ such that $\varphi_{n} \rightarrow \varphi$ and $A_{p} \varphi_{n} \rightarrow \varphi$ in $L^{p}(\Omega)$. (For example, we can take $\varphi_{n}=$ $\left(A_{p}+k+1\right)^{-1} \psi_{n}=\left(A_{q}+k+1\right)^{-1} \psi_{n}$ where $\left\{\psi_{n}\right\}$ is a sequence in $L^{q}(\Omega)$ with $\psi_{n} \rightarrow\left(A_{p}+k+1\right) \varphi$ in $L^{p}(\Omega)$ and $k$ is chosen so that Proposition 4.2 holds.) Let $u(t)=S(t) \varphi$ and $u_{n}=S(t) \varphi_{n}$ be constructed from $\varphi$ and $\varphi_{n}$ by Proposition 4.3. Since the $S(t)$ are Lipschitz maps, $u_{n}(t)$ converges to $u(t)$ in $L^{p}(\Omega)$. By Lemma 4.5, $\left\{\left(T_{q}+k\right)^{\alpha} u_{n}(t)\right\}$ is a bounded sequence in $L^{q}(\Omega)$, for fixed $t>0$. Since $L^{q}(\Omega)$ is reflexive, there is a subsequence $\left\{u_{n_{j}}(t)\right\}$ such that $\left\{u_{n_{j}}(t)\right\}$ and $\left\{\left(T_{q}+k\right)^{\alpha} u_{n_{j}}(t)\right\}$ converge weakly in $L^{q}(\Omega)$, say $u_{n_{j}}(t) \rightarrow v$ and $\left(T_{q}+k\right)^{\alpha} u_{n_{j}}(t) \rightarrow w$ weakly in $L^{q}(\Omega)$. It follows that $\left\{\left(u_{n_{j}}(t),\left(T_{q}+k\right)^{\alpha} u_{n_{j}}(t)\right)\right\}$ converges weakly to $(v, w)$ in $L^{q}(\Omega) \times L^{q}(\Omega)$. Since the graph of $\left(T_{q}+k\right)^{\alpha}$ is closed (and, hence weakly closed), $v \in D\left(\left(T_{q}+k\right)^{\alpha}\right)$. However, we must have $u(t)=v$, since $\left(u_{n_{j}}(t), \psi\right) \rightarrow(u(t), \psi)$ and $\left(u_{n_{j}}(t), \psi\right) \rightarrow(v, \psi)$ for every test function $\psi$. It follows that $u(t) \in D\left(\left(T_{q}+k\right)^{\alpha}\right)$. From part (3) of Proposition 4.3 it follows that $u:(t, \infty) \rightarrow W^{2, q}(\Omega)$ is analytic. Since $t>0$ is arbitrary, this proves the theorem.

\section{REFERENCES}

1. S. Agmon, A. Douglis and L. Nirenberg, Estimates near the boundary for solutions of elliptic partial differential equations satisfying general boundary conditions, $I$, Comm. Pure Appl. Math., 12 (1959), 623-727.

2. H. Brézis, Propriétés regularisantes de certains semi-groups non linéaires, Israel J. Math., 9 (1971), 513-534.

3. H. Brézis, M. G. Crandall and A. Pazy, Perturbations of nonlinear maximal monotone sets in Banach space, Comm. Pure Appl. Math., 23 (1970), 123-144.

4. H. Brézis and W. Strauss, Semi-linear second-order elliptic equations in $L^{1}$, J. Math. Soc. Japan, to appear.

5. M. Crandall and T. Liggett, Generation of semi-groups of nonlinear transformations on general Banach spaces, Amer. J. Math., 93 (1971), 265-298.

6. M. Crandall and A. Pazy, Semi-groups of nonlinear contractions and dissipative sets, J. Functional Analysis, 3 (1969), 376-418.

7. G. Da Prato, Somme d'applications non linéaires, Symposia Mathematica VII, 1 st. Naz. di Alta Mat., Academic Press, London, 1971.

8. A. Friedman, Partial Differential Equations, Holt, Rinehart and Winston, New York, 1969.

9. H. Fujita and T. Kato, On the Navier-Stokes initial value problem, I, Arch. Rational Mech. Anal., 16 (1964), 269-315.

10. D. Henry, Geometric theory of semilinear parabolic equations, mimeographed notes, University of Kentucky, 1974.

11. E. Hille and R. S. Phillips, Functional analysis and semi-groups, revised, ed., Amer. Math. Soc. Colloq. Publ., Vol. 31, Amer. Math. Soc., Providence, 1957.

12. T. Kato, Perturbation theory for linear operators, Die Grundlehren der math. Wissenschaften, Band 132, Springer-Verlag, New York, 1966.

13. - Nonlinear semigroups and evolution equations, J. Math. Soc. Japan, 19 (1967), 508-520. 
14. T. Kato, Accretive operators and nonlinear evolution equations in Banach spaces, Proc. Symp. Pure Math. 18, Part I, Amer. Math. Soc., Providence, 138-161 (1968).

15. Y. Kōmura, Nonlinear semi-groups in Hilbert space, J. Math. Soc. Japan, 19 (1967), 493-507.

16. — Differentiability of nonlinear semigroups, J. Math. Soc. Japan, 21 (1969), 375-402.

17. Y. Konishi, Some examples of nonlinear semi-groups in Banach lattices, J. Fac. Sci. Univ. Tokyo, 18 (1972), 537-543.

18. S. G. Krein, Linear differential equations in a Banach space, Translations of Mathematical Monographs, Vol. 29, Amer. Math. Soc., Providence, 1971.

19. K. Masuda, On the analyticity and the unique continuation theorem for solutions of the Navier-Stokes equation, Proc. Japan Acad., 43 (1967), 827-832.

20. I. Miyadera, Some remarks on semigroups of nonlinear operators, Tôhoku Math. J., 23 (1971), 245-258.

21. J. W. Neuberger, Existence of a spectrum for nonlinear transformations, Pacific J. Math., 31 (1969), 157-159.

22. S. Ōuchi, On the analyticity in time of solutions of initial boundary value problems for semilinear parabolic differential equations with monotone nonlinearity, J. Fac. Sci. Univ. Tokyo, 20 (1974), 19-41.

23. P. E. Sobolevskii, Equations of parabolic type in a Banach space, Trudy Moscow Mat. Obšč. 10 (1961), 297-350; English transl., Amer. Math. Soc. Transl. (2) 49 (1965), $1-62$.

24. K. Yosida, Functional analysis, 2nd ed., Die Grundlehren der math. Wissenschaften, Band 123, Springer-Verlag, New York, 1968.

Received February 27, 1974 and in revised form March 3, 1975.

UNIVERSITY OF KENTUCKY 



\section{PACIFIC JOURNAL OF MATHEMATICS}

\section{EDITORS}

RICHARD ARENS (Managing Editor)

University of California

Los Angeles, California 90024

\section{J. DugundJI}

Department of Mathematics University of Southern California Los Angeles, California 90007

D. Gilbarg and J. Milgram

Stanford University

Stanford, California 94305
University of Washington Seattle, Washington 98105

\section{ASSOCIATE EDITORS}
E. F. BECKENBACH
B. H. NeumanN
F. WolF
K. YoShIDA

\section{SUPPORTING INSTITUTIONS}

\author{
UNIVERSITY OF SOUTHERN CALIFORNIA \\ STANFORD UNIVERSITY \\ UNIVERSITY OF TOKYO \\ UNIVERSITY OF UTAH \\ WASHINGTON STATE UNIVERSITY \\ UNIVERSITY OF WASHINGTON \\ $\stackrel{*}{*} \stackrel{*}{*} \stackrel{*}{ }{ }^{*}$ AMERICAN MATHEMATICAL SOCIETY
}

The Supporting Institutions listed above contribute to the cost of publication of this Journal, but they are not owners or publishers and have no responsibility for its content or policies.

Mathematical papers intended for publication in the Pacific Journal of Mathematics should be in typed form or offset-reproduced, (not dittoed), double spaced with large margins. Underline Greek letters in red, German in green, and script in blue. The first paragraph or two must be capable of being used separately as a synopsis of the entire paper. Items of the bibliography should not be cited there unless absolutely necessary, in which case they must be identified by author and Journal, rather than by item number. Manuscripts, in triplicate, may be sent to any one of the editors. Please classify according to the scheme of Math. Reviews, Index to Vol. 39. All other communications should be addressed to the managing editor, or Elaine Barth, University of California, Los Angeles, California, 90024.

The Pacific Journal of Mathematics expects the author's institution to pay page charges, and reserves the right to delay publication for nonpayment of charges in case of financial emergency.

100 reprints are provided free for each article, only if page charges have been substantially paid. Additional copies may be obtained at cost in multiples of 50 .

The Pacific Journal of Mathematics is issued monthly as of January 1966. Regular subscription rate: $\$ 72.00$ a year (6 Vols., 12 issues). Special rate: $\$ 36.00$ a year to individual members of supporting institutions.

Subscriptions, orders for back numbers, and changes of address should be sent to Pacific Journal of Mathematics, 103 Highland Boulevard, Berkeley, California, 94708.

\section{PUBLISHED BY PACIFIC JOURNAL OF MATHEMATICS, A NON-PROFIT CORPORATION}

Printed at Kokusai Bunken Insatsusha (International Academic Printing Co., Ltd.), 270, 3-chome Totsuka-cho, Shinjuku-ku, Tokyo 160, Japan.

\section{Copyright (C) 1975 by Pacific Journal of Mathematics} Manufactured and first issued in Japan 


\section{Pacific Journal of Mathematics}

\section{Vol. 57, No. $2 \quad$ February, 1975}

Norman Larrabee Alling, On Cauchy's theorem for real algebraic curves with boundary .......

Daniel D. Anderson, A remark on the lattice of ideals of a Prüfer domain ..................

Dennis Neal Barr and Peter D. Miletta, A necessary and sufficient condition for uniqueness of

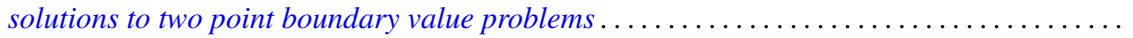

Ladislav Beran, On solvability of generalized orthomodular lattices . . . . . . . . . . ........

L. Carlitz, A three-term relation for some sums related to Dedekind sums . . . . . . . . . .....

Arthur Herbert Copeland, Jr. and Albert Oscar Shar, Images and pre-images of localization

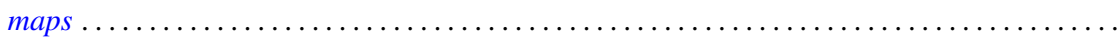

G. G. Dandapat, John L. Hunsucker and Carl Pomerance, Some new results on odd perfect

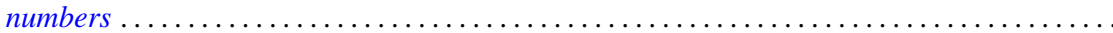

M. Edelstein and L. Keener, Characterizations of infinite-dimensional and nonreflexive

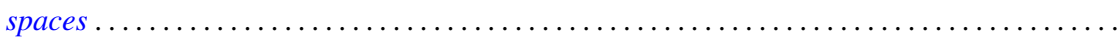

Francis James Flanigan, On Levi factors of derivation algebras and the radical embedding

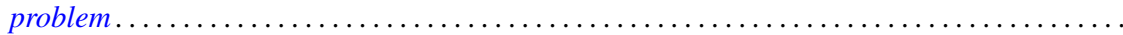

Harvey Friedman, Provable equality in primitive recursive arithmetic with and without

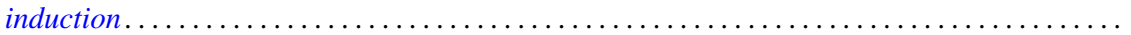

Joseph Braucher Fugate and Lee K. Mohler, The fixed point property for tree-like continua with

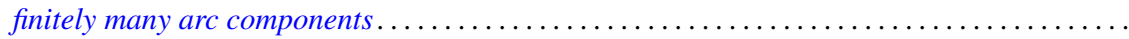

John Norman Ginsburg and Victor Harold Saks, Some applications of ultrafilters in

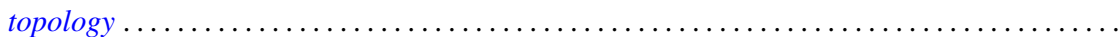

Arjun K. Gupta, Generalisation of a "square" functional equation .....................

Thomas Lee Hayden and Frank Jones Massey, Nonlinear holomorphic semigroups ..........

V. Kannan and Thekkedath Thrivikraman, Lattices of Hausdorff compactifications of a locally

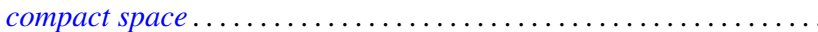

J. E. Kerlin and Wilfred Dennis Pepe, Norm decreasing homomorphisms between group

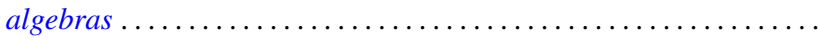

Young K. Kwon, Behavior of $\Phi$-bounded harmonic functions at the Wiener boundary ...

Richard Arthur Levaro, Projective quasi-coherent sheaves of modules .

Chung Lin, Rearranging Fourier transforms on groups...........................

David Lowell Lovelady, An asymptotic analysis of an odd order linear differential equation . . 4475

Jerry Malzan, On groups with a single involution .......................... 481

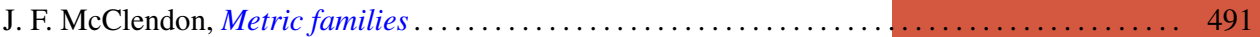

Carl Pomerance, On multiply perfect numbers with a special property .

Mohan S. Putcha and Adil Mohamed Yaqub, Polynomial constraints for finiteness of semisimple rings. .

Calvin R. Putnam, Hyponormal contractions and strong power convergence . . . . . . . . . 531

Douglas Conner Ravenel, Multiplicative operations in $\mathrm{BP} * \mathrm{BP} \ldots \ldots \ldots \ldots \ldots \ldots \ldots \ldots \ldots .539$

Judith Roitman, Attaining the spread at cardinals which are not strong limits . . . . . . . . . 545

Kazuyuki Saitô, Groups of *-automorphisms and invariant maps of von Neumann algebras . . . 553

Brian Kirkwood Schmidt, Homotopy invariance of contravariant functors acting on smooth

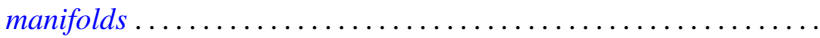

Kenneth Barry Stolarsky, The sum of the distances to $N$ points on a sphere.

Mark Lawrence Teply, Semiprime rings with the singular splitting property.

J. Pelham Thomas, Maximal connected Hausdorff spaces..............

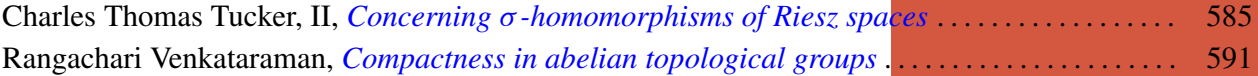

William Charles Waterhouse, Basically bounded functors and flat sheaves . . . . . . . . . . . 597

David Westreich, Bifurcation of operator equations with unbounded linearized part ......... 611

William Robin Zame, Extendibility, boundedness and sequential convergence in spaces of 